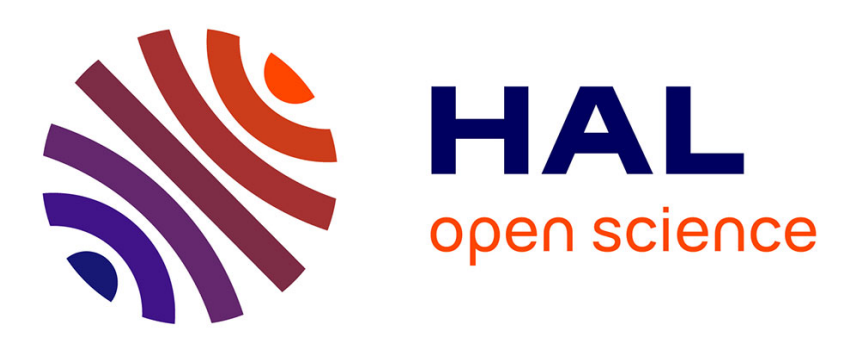

\title{
A comparison of damage detection methods applied to civil engineering structures
}

\author{
Szymon Gres, Palle Andersen, Rasmus Johan Johansen, Martin Dalgaard \\ Ulriksen, Lars Damkilde
}

\section{- To cite this version:}

Szymon Gres, Palle Andersen, Rasmus Johan Johansen, Martin Dalgaard Ulriksen, Lars Damkilde. A comparison of damage detection methods applied to civil engineering structures. EVACES 2017 Experimental Vibration Analysis of Civil Engineering Structures, Jul 2017, San Diego, United States. 10.1007/978-3-319-67443-8_26. hal-02421402

\section{HAL Id: hal-02421402 \\ https://hal.inria.fr/hal-02421402}

Submitted on 20 Dec 2019

HAL is a multi-disciplinary open access archive for the deposit and dissemination of scientific research documents, whether they are published or not. The documents may come from teaching and research institutions in France or abroad, or from public or private research centers.
L'archive ouverte pluridisciplinaire HAL, est destinée au dépôt et à la diffusion de documents scientifiques de niveau recherche, publiés ou non, émanant des établissements d'enseignement et de recherche français ou étrangers, des laboratoires publics ou privés. 


\title{
A comparison of damage detection methods applied to civil engineering structures
}

\author{
Szymon Gres ${ }^{1}$, Palle Andersen ${ }^{2}$, Rasmus Johan Johansen ${ }^{3}$, Martin Dalgaard Ulriksen ${ }^{3}$, Lars Damkilde ${ }^{4}$ \\ ${ }^{1}$ E.Ph.D. Student, Department of Structural Engineering, Aalborg University, Universal Foundation A/S, Aalborg, Denmark. \\ ${ }^{2}$ Managing director, Ph.D., Structural Vibration Solutions, Aalborg, Denmark. \\ ${ }^{3} \mathrm{Ph}$.D. Student, Department of Structural and Offshore Engineering, Aalborg University, Esbjerg, Denmark \\ ${ }^{4}$ Professor, Department of Structural Engineering, Aalborg University, Aalborg, Denmark
}

\begin{abstract}
The early damage detection, among localization and quantification methods, is crucial for the in-time repairs and cost-optimized maintenance plans of civil engineering structures. Preferably, the damage detection is performed by use of the output vibration data, hereby avoiding modal identification of the structure. The most of the work within the field assumes that the unmeasured excitation signal is time-invariant with a constant covariance, which is hardly achieved in practice. In this paper, we present a comparison of a new Mahalanobis distance-based damage detection method with the well-known subspace-based damage detection algorithms robust to the changes in the excitation covariance. The joint features of the methods are concluded in a control chart. The described methodology is implemented in ARTeMIS- a software for health monitoring and operational modal analysis. The performances of the methods are evaluated both on the numerical simulations and a full-scale experimental example---namely data obtained from the Dogna Bridge---using the output ambient vibration signals. The results reveal a positive capability of the damage detection from tested methods, with a less computational complexity of the Mahalanobis distance-based approach. The control chart presents a comprehensive overview of the progressively damaged structure.
\end{abstract}

Keywords: Structural health monitoring, ambient excitation, damage detection, control chart-based algorithm fusion.

\section{INTRODUCTION}

In the field of damage diagnosis, the vibration-based methods are effective in the detection of a small deviations solely based on the operational data from the structure [1]. The practical aspect of using only the output measurements cause several difficulties due to the variations in the ambient excitation, which can be due to variability in environmental conditions, for example, wind, temperature and precipitation, and operational conditions, [7]. This issue is addressed in several detection techniques available in the literature [7-12] and effectively implemented for the condition based maintenance and the early damage detection [10]. A general review of the damage detection strategies is presented in [2-3].

The concept of vibration-based damage detection relates to identification of the deviations in the damage-sensitive features of the collected output data. The deviations are defined by a relative comparison of the reference and operational states of the system. The commonly used features for the detection are the modal parameters (natural frequencies, mode shapes and damping ratios) identified from the data. However, some research questions the use of the modal parameters, arguing that the modal data itself is not sensitive enough to detect the local faults [3], especially when in practice the structure is excited by low-frequency inputs. One workaround for the system identification when estimating the features is to use the statistical properties of the data. The statistical methods use the probability distributions of the deviations, which differ between the damaged and the undamaged states indicating the faults in the system.

The focus of this work is twofold. One is to present a simple statistical approach for the damage detection based on the Mahalanobis distance featured with the Hankel matrices. The distance metric is calculated on the output vibration data processed in the framework similar to subspace-based methods. The test is robust towards the changes in the excitation covariance. The damage is detected by the deviations of the distance from the reference test state. The results are compared with the xi2-test build on the residual from the subspace-based methods [9-13]. The second objective is to present a complete, practical overview of the damage indicators combined from the both methods in the Hotelling control chart [18].

The methods are tested both on the numerical simulations and the full-scale bridge. The academic example is a coupled springmass system, excited by white noise of random variance. The comparison is concluded by use of data from the full-scale Dogna Bridge, Italy. The bridge is artificially progressively damaged and excited by the wind.

The structure of the paper is as follows. The basic principles of the robust subspace-based damage detection method and the methodology for the Mahalanobis distance calculated on the Hankel matrices is presented in Section 2. The two test examples 
are described in Section 3. Both the comparison and joint performance of the methods on the numerical and full scale detection cases are presented in Section 4. The results are concluded in Section 5.

\section{METHODOLOGY}

In this section we recall the basic principles of the subspace-based damage detection [12] and present the algorithm for the Mahalanobis distance-based damage detection. Both methods are data-driven.

\section{SUBSPACE-BASED DAMAGE DETECION}

The damage detection consists of monitoring the deviations of the system from its reference state, characterized by some nominal property repeatable for every healthy conditions. The deviations are defined by a residual that differs between the healthy and the damaged states [13]. Consider the discrete state space model

$$
\begin{gathered}
\mathbf{x}_{\mathrm{k}+1}=\mathbf{F} \mathbf{x}_{\mathrm{k}}+\mathbf{v}_{\mathrm{k}} \\
\mathbf{y}_{\mathrm{k}}=\mathbf{H} \mathbf{x}_{\mathrm{k}}+\mathbf{w}_{\mathrm{k}}
\end{gathered}
$$

with the state transition matrix $\mathbf{F} \in R^{n x n}$, the observation matrix $\mathbf{H} \in R^{r x n}$, the states $\mathbf{x}_{\mathbf{k}} \in R^{n}$ and the outputs $\mathbf{y}_{\mathbf{k}} \in R^{r}$, where $r$ is the number of the sensors and $n$ denotes the order of the system, [6]. The unmeasured white noise input $\mathbf{v}_{\mathbf{k}}$ drives the dynamics of the system and $\mathbf{w}_{\mathbf{k}}$ is the measurement noise. Any perturbation in the structural properties of the system, manifested in the stiffness or mass, leads to deviations in the state matrices and are subsequently reflected in $\mathbf{y}_{\mathbf{k}}$. As a consequence, damage changes the eigenstructure of the state space model. Hence, the damage-sensitive system property relates to the $\mathbf{F}$ and $\mathbf{H}$ matrices.

Let $\boldsymbol{\Sigma}_{s}=\mathrm{E}\left(\mathbf{x}_{\mathrm{k}+1} \mathbf{x}_{\mathrm{k}+1}^{\mathrm{T}}\right)$ be the state covariance matrix and $\mathbf{G}=\mathrm{E}\left(\mathbf{x}_{\mathrm{k}+1} \mathbf{y}_{\mathrm{k}}^{\mathbf{T}}\right)=\mathbf{F} \boldsymbol{\Sigma}_{\mathrm{s}} \mathbf{H}^{T}$ the cross-covariance between the states and the outputs [6]. The output covariance yields $\boldsymbol{\Lambda}_{\mathrm{i}}=\mathrm{E}\left(\mathbf{y}_{\mathrm{k}+\mathrm{i}} \mathbf{y}_{\mathrm{k}}^{\mathbf{T}}\right)=\mathbf{H} \mathbf{F}^{\mathrm{i}-1} \mathbf{G}$ and can be structured in the block-Hankel matrix

$$
\mathbf{H}_{p+1, q}=\left[\begin{array}{cllc}
\boldsymbol{\Lambda}_{1} & \boldsymbol{\Lambda}_{2} & \ldots & \boldsymbol{\Lambda}_{q} \\
\boldsymbol{\Lambda}_{2} & \boldsymbol{\Lambda}_{3} & \ldots & \boldsymbol{\Lambda}_{q+1} \\
\vdots & \vdots & \ddots & \vdots \\
\boldsymbol{\Lambda}_{p+1} & \boldsymbol{\Lambda}_{p+2} & \ldots & \boldsymbol{\Lambda}_{p+q}
\end{array}\right]=\operatorname{Hank}\left(\boldsymbol{\Lambda}_{\mathrm{i}}\right) .
$$

$\mathbf{H}_{p+1, q} \in R^{(p+1) r x q r}$ where $p$ and $q$ are parameters such $q=p+1$. The $\mathbf{H}_{p+1, q}$ can be factorized into the observability and controllability matrices such

$$
\mathbf{H}_{p+1, q}=\mathbf{0}_{p+1, q} \mathbf{C}_{q}
$$

where the observability matrix, $\mathbf{0}_{p+1, q} \in R^{(p+1) r \times n}$, and controllability matrix, $\mathbf{C}_{q} \in R^{n \times q r}$, are given by

$$
\mathbf{O}_{p+1, q}=\left[\begin{array}{c}
\mathbf{H} \\
\mathbf{H F} \\
\vdots \\
\mathbf{H} \mathbf{F}^{p}
\end{array}\right], \mathbf{C}_{q}=\left[\begin{array}{llll}
\mathbf{G} & \mathbf{F G} & \ldots & \mathbf{F}^{q-1} \mathbf{G}
\end{array}\right] .
$$

The subspace-based fault detection test detects if the residual of the characteristic property of the reference (healthy) state of the system based on $\mathbf{0}_{p+1, q}$, or subsequently $\mathbf{H}_{p+1, q}$ (Eq. 5), is significantly different from zero. Consider the space, or a property of the reference state, where

$$
\mathbf{S}^{T} \widehat{\mathbf{H}}_{p+1, q} \approx 0
$$

$\widehat{\mathbf{H}}_{p+1, q}$ is the empirical output block-Hankel matrix and $\mathbf{S}$ is the left kernel $\mathbf{U}_{0}$ of the reference state $\widehat{\mathbf{H}}_{p+1, q}$, hence

$$
\widehat{\mathbf{H}}_{p+1, q}{ }^{r e f}=\left[\begin{array}{ll}
\mathbf{U}_{1} & \mathbf{U}_{0}
\end{array}\right]\left[\begin{array}{cc}
\Delta_{1} & 0 \\
0 & \Delta_{0}
\end{array}\right]\left[\begin{array}{l}
\mathbf{V}_{1}^{T} \\
\mathbf{V}_{0}^{T}
\end{array}\right]
$$

where $\Delta_{1}$ contains non-zero singular values. The reference and the subsequently tested states share the same left null space only when no damage occurs. Bearing that in mind, the residual vector is defined as 


$$
\xi=\sqrt{N} \operatorname{vec}\left(\mathbf{S}^{T} \widehat{\mathbf{H}}_{p+1, q}\right) .
$$

Note that the excitation of the system is ambient, which leads to the changes in the cross-covariance matrix, $\mathbf{G}$, (recall $\mathbf{G}=$ $\left.\mathrm{E}\left(\mathbf{x}_{\mathrm{k}+1} \mathbf{y}_{\mathrm{k}}^{\mathrm{T}}\right)=\mathbf{F} \boldsymbol{\Sigma}_{\mathrm{s}} \mathbf{H}^{T}\right)$, and consequently in the output block Hankel matrix $\widehat{\mathbf{H}}_{p+1, q}$. That results in non-zero residuals (Eq. 7) between the healthy states. To avoid that, [13] use the fact that $\widehat{\mathbf{H}}_{p+1, q}$ shares the same null space with its principal left singular vectors $\mathbf{U}_{1}$, so for each healthy state it holds that $\mathbf{S}^{T} \mathbf{U}_{1} \approx 0$. Matrix $\mathbf{U}_{1}$ contains $n$ independent column vectors that spans the column space of $\widehat{\mathbf{H}}_{p+1, q}$ and are principal directions of the data, invariant to the change in excitation covariance [19]. The robust residual is defined as

$$
\xi=\sqrt{N} \operatorname{vec}\left(\mathbf{S}^{T} \mathbf{U}_{1}\right)
$$

which is tested for being significantly different from zero by use of the $\chi^{2}$-test

$$
\chi_{\xi}^{2}=\xi^{T} \boldsymbol{\Sigma}_{\xi}^{-1} \xi
$$

where $\boldsymbol{\Sigma}_{\xi}$ is the empirical covariance of the residual calculated as in [12].

\section{MAHALANOBIS DISTANCE-BASED DAMAGE DETECION}

The Mahalanobis distance, MD, is a metric used in the multivariate statistics to calculate the distance between a point and a distribution. The formulation of the metric takes into account the correlations between different data dimensions by the covariance matrix of the data. For the multivariate normally distributed variables the squared MD follows the $\chi^{2}$-distribution [20]. One of its practical uses is detection of outliers [16] and recently detection of structural damages based on the transmissibility functions or AR-coefficients as a feature [14-15].

The square Mahalanobis metric of the observations in the data vector $\mathbf{x}_{i}$, from the reference data vector with the sample mean $\boldsymbol{\mu}$ and the covariance matrix $\boldsymbol{\Sigma}$ is defined as

$$
M D_{i}=\left(\mathbf{x}_{i}-\boldsymbol{\mu}\right)^{T} \boldsymbol{\Sigma}^{-1}\left(\mathbf{x}_{i}-\boldsymbol{\mu}\right)
$$

In this paper the Mahalanobis distance is calculated on a damage sensitive parameter of the system between a reference and damaged states and used directly as a damage indicator. Proposed metric is robust towards the variations of the excitation covariance hence can be used with the operational measurements. Consider $m$ reference data sets and $i$ tested states, so that

$$
\begin{gathered}
M D_{i}<T_{m} \rightarrow \text { healthy } \\
M D_{i}>T_{m} \rightarrow \text { damaged }
\end{gathered}
$$

where $T_{m}$ is a threshold calculated for the reference state. The MD increases as the system is subjected to any change that affects its vibration characteristics. Here, we consider the empirical block-Hankel matrices with the output correlations as the input for the damage detection test. The modified version of the squared MD featured with the Hankel matrices is defined as

$$
M D_{i}=\operatorname{vec}\left(\widehat{\mathbf{H}}_{p+1, q}^{c o r r}\right)^{T}\left(\boldsymbol{\Sigma}_{\widehat{\mathbf{H}}_{p+1, q}^{c o r r, r e f}}\right)^{-1} \operatorname{vec}\left(\widehat{\mathbf{H}}_{p+1, q}^{c o r r}\right)
$$

Let $Y \in R^{r x m N}$ where $m$ is the number of the reference sets and $N$ is the length of one data set. The formulation of the empirical block-Hankel matrix yields

$$
\widehat{\mathbf{C}}_{i}=\frac{1}{m N} \sum_{k=1}^{m N} \frac{\mathbf{Y}_{k} \mathbf{Y}_{k-i}^{T}}{\sigma_{k} \sigma_{k-i}} ; \widehat{\mathbf{H}}_{p+1, q}^{c o r r, r e f}=\operatorname{Hank}\left(\widehat{\mathbf{C}}_{i}\right) .
$$

Consequently, $\widehat{\mathbf{H}}_{p+1, q}^{\text {corr }}$ is determined using Eq. 12 for each tested data set with $m=1$. The covariance of the empirical vectorized Hankel matrix is estimated from the covariance of the sample mean with the methodology recalled from [12]. The threshold above which the data set is considered damaged is defined as one standard deviation above the mean value of the reference state, that theoretically should approach zero. 


\section{TEST CASES}

Methods described in the Section 2 are tested with a both numerical example of a simple system excited by the white noise of random variance and a full scale progressively damaged bridge excited by the wind.

\section{SIMPLE SYSTEM SIMULATION}

For the numerical testing we considered a coupled spring-mass system with 15 DOF, Figure 1. Output accelerations are simulated using white noise input of variance taken randomly from the normally distributed vector in between [1 10], acting on the last DOF. The data is recorded in all DOFs along the system. To account for the noise in the measurements 3\%, 10\% and $30 \%$ of white noise is added on top of the response signals.

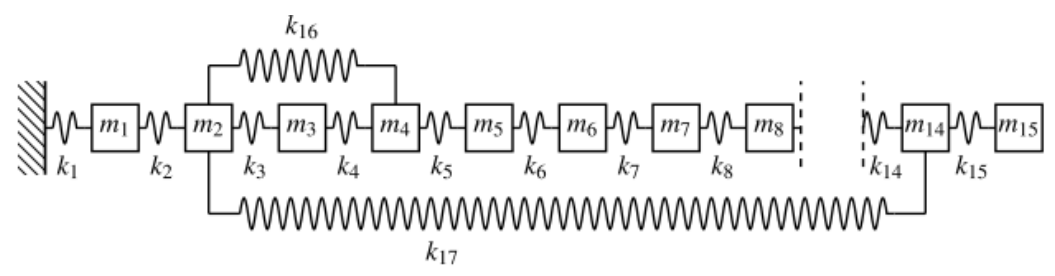

Figure 1. Simple system scheme.

The damage is simulated as a progressive reduction in the stiffness of the $8^{\text {th }}$ spring by $2 \%, 5 \%, 10 \%$ and $30 \%$. The sampling frequency is $50 \mathrm{~Hz}$. The system is exited for $1000 \mathrm{~s}$ in blocks of 50 data sets for each simulated case. That results in 250 data records per sensor per noise level with a random variance in between each set.

\section{DOGNA BRIDGE}

The Dogna bridge, Figure 2, crosses the River Fella and connects the villages of Crivera and Valdogna (Dogna) in Friuli Venezia Giulia, a region located in the North East of Italy.

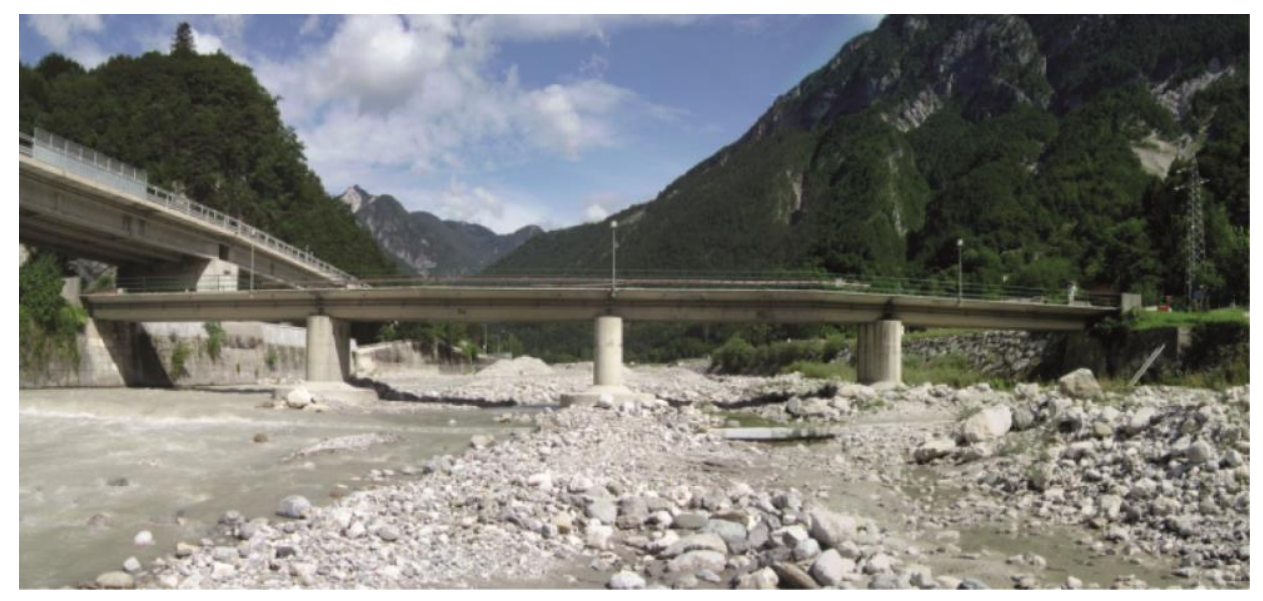

Figure 2. Dogna bridge, Italy, after [17].

The bridge is a four-span, single-lane concrete bridge. The span is $16 \mathrm{~m}$, with the $4 \mathrm{~m}$ lane. The deck is made of a $0.18 \mathrm{~m}$ reinforced concrete (RC) slab, supported by a three longitudinal simply supported RC beams with a rectangular cross-section $0.35 \times 1.20 \mathrm{~m}$. The beams are connected to the supports with the rectangular RC diaphragms $0.3 \times 0.7 \mathrm{~m}$. The piers are a RC walls approx. $1.5 \mathrm{~m}$ thick, $4.5 \mathrm{~m}$ deep, and $3.6 \mathrm{~m}$ high. The pylons are fixed in a piled foundation that consists of a $1 \mathrm{~m}$ thick RC slab supported by a drilled RC piles of $1 \mathrm{~m}$ in diameter and $18 \mathrm{~m}$ in length.

For a traffic safety reasons, the Dogna bridge was demolished on May 2008 and has been replaced by a new bridge built about 200m downstream. A test campaign was carried out from April 02 to April 11 2008, and consisted of a series of tests progressively damaging one of the bridge spans. The tests were carried out under similar environmental conditions so the influence of temperature and humidity on the structure is insignificant. Figure 3 shows the artificially damaged bridge span during the tests. 


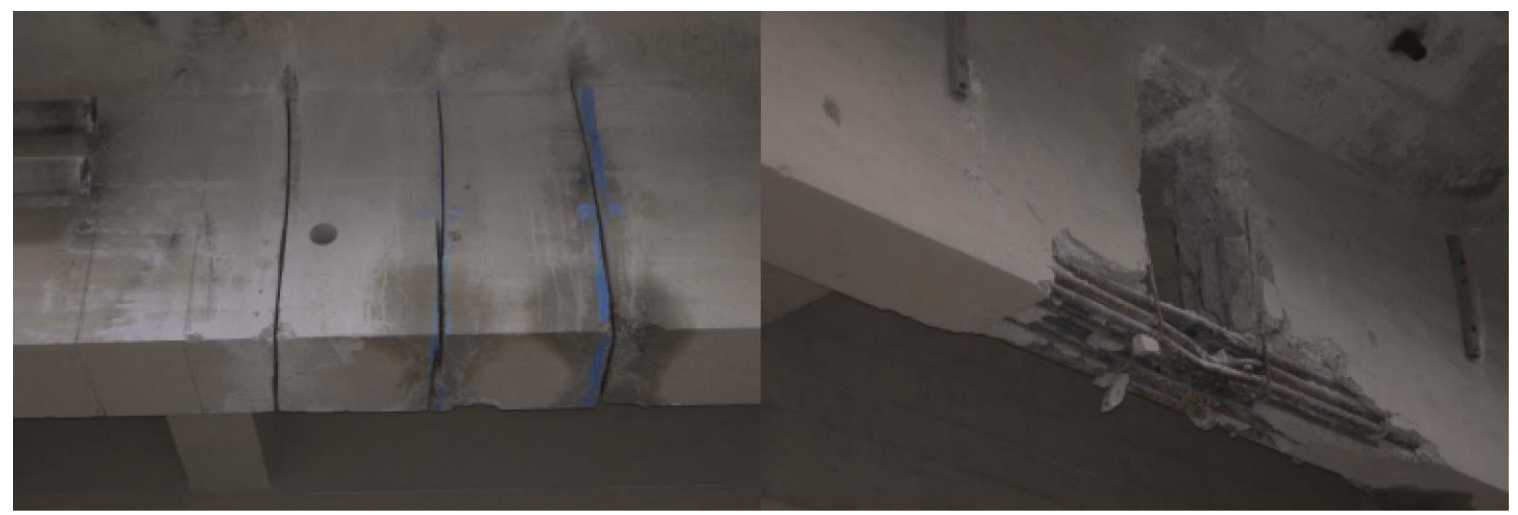

Figure 3. Artificially damaged beams. The cuts introduced progressively during the tests, along with the damage in the centerline of the span. After [17]

The bridge was equipped with 10 accelerometers mounted on its deck. The samples were taken with the frequency of $400 \mathrm{~Hz}$. The measurement campaign lasted 50 minutes in total while the reference state was measured for 20 minutes. The damaged was introduced in approx. three blocks: the first cut, the second cut and the third cut with the damaged center span.

\section{RESULTS}

In this section we compare the results from the classical subspace-based damage detection implemented in ARTeMIS - software for operational modal analysis and health monitoring [17] with the Mahalanobis based damage detection described in the paper. The results for the each test case are concluded in the control chart.

Note again, that both test cases are excited by a ambient, unmeasured load with changing variance.

\section{SIMPLE SYSTEM SIMULATION}

The damage is detected for the three noise levels present in the response of the simple system. The 30 out of 50 healthy data sets are used to calculate the reference state. The thresholds for both of methods are defined as a number of standard deviations above the mean metric of the reference state. One standard deviation for the unsafe zone, and two standard deviations for the damaged zone.

Figure 4 and Figure 5 illustrate the comparison of the damage indicators for the 3\% and 30\% of white noise it the response data. The percentage of the detected damage sets with respect to the simulation case and degree of the damage is summarized in the Table 1.
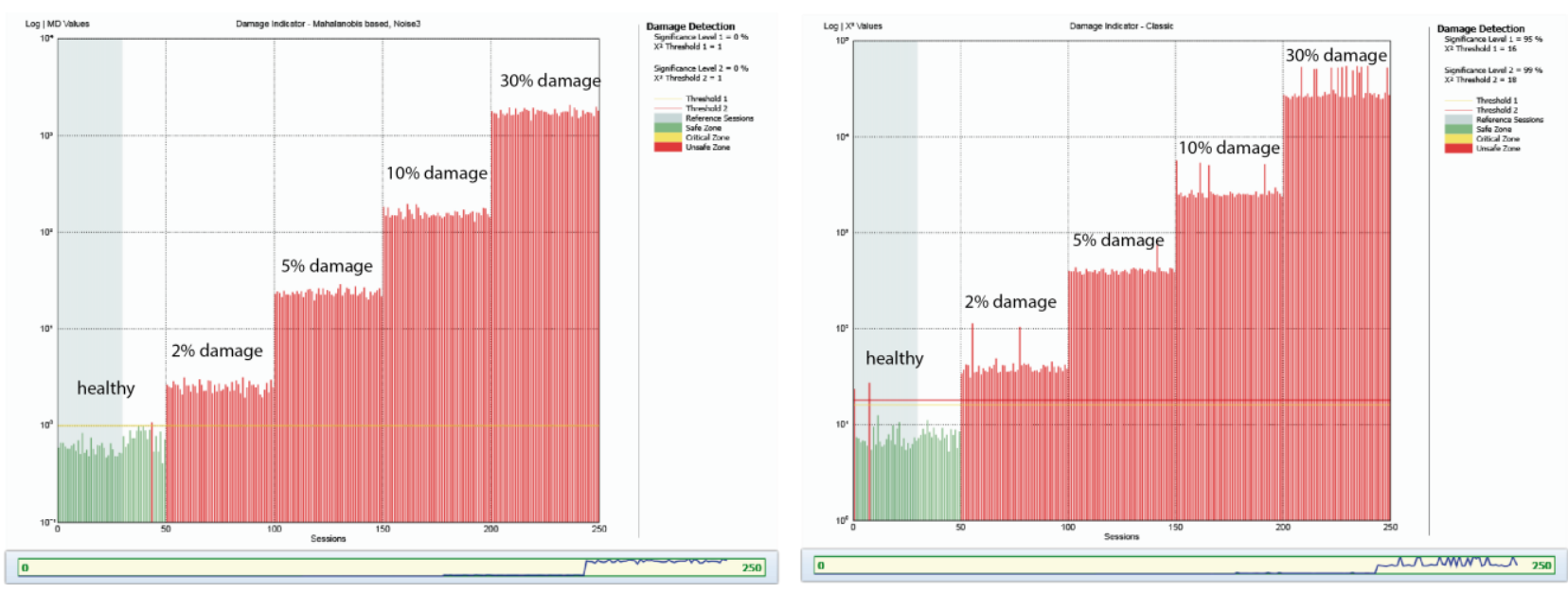

Figure 4. Damage detection in simple system. Case-3\% of noise in the response. Damage indicators for the Mahalanobisbased (left) and robust subspace-based (right).

Both methods detect each degree of the damage present in the response data containing $3 \%$ of white noise, disregarding the variance of the excitation, Figure 4. While increasing the noise level to 30\%, the Mahalanobis-based approach perform more accurate in detecting the smaller deviations, see Figure 5 and Table 1. The control chart, created for the 30\% noise case, gives ability to detect all the 5\% damage and half of $2 \%$ damaged sets, what was not possible with a single method, see Figure 6. 

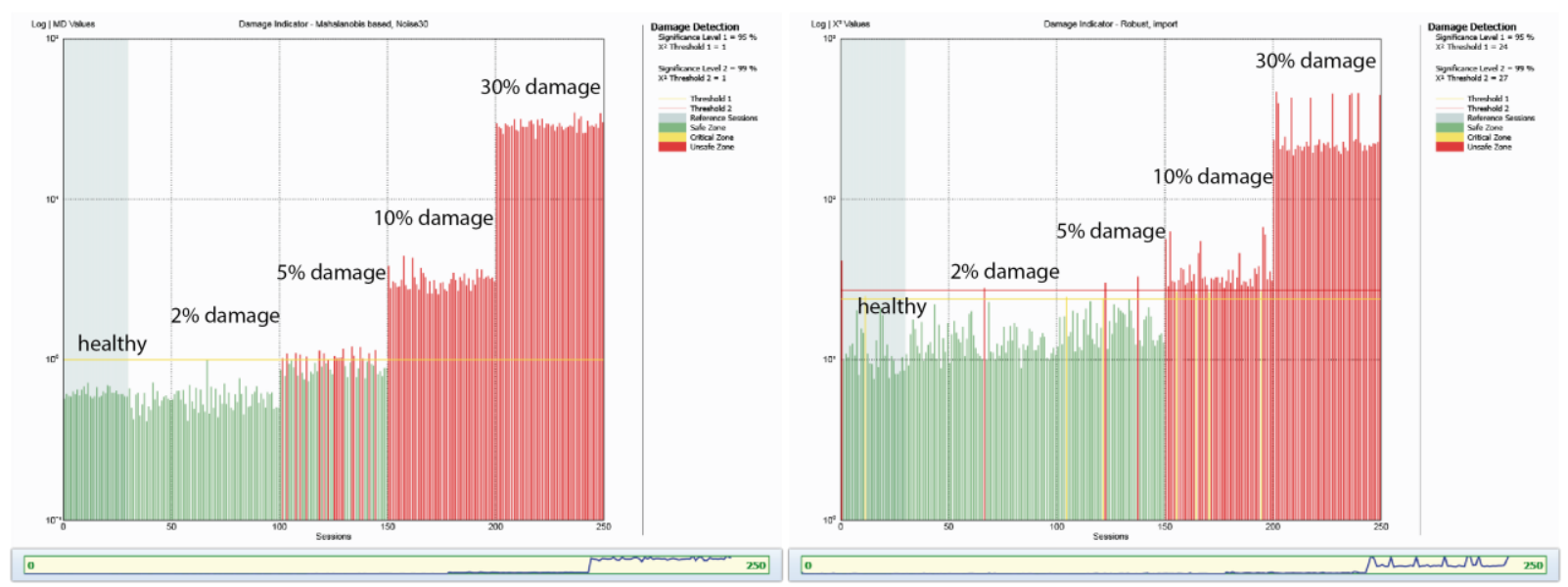

Figure 5. Damage detection in simple system. Case-30\% of noise in the response. Damage indicators for the Mahalanobisbased (left) and robust subspace-based (right).

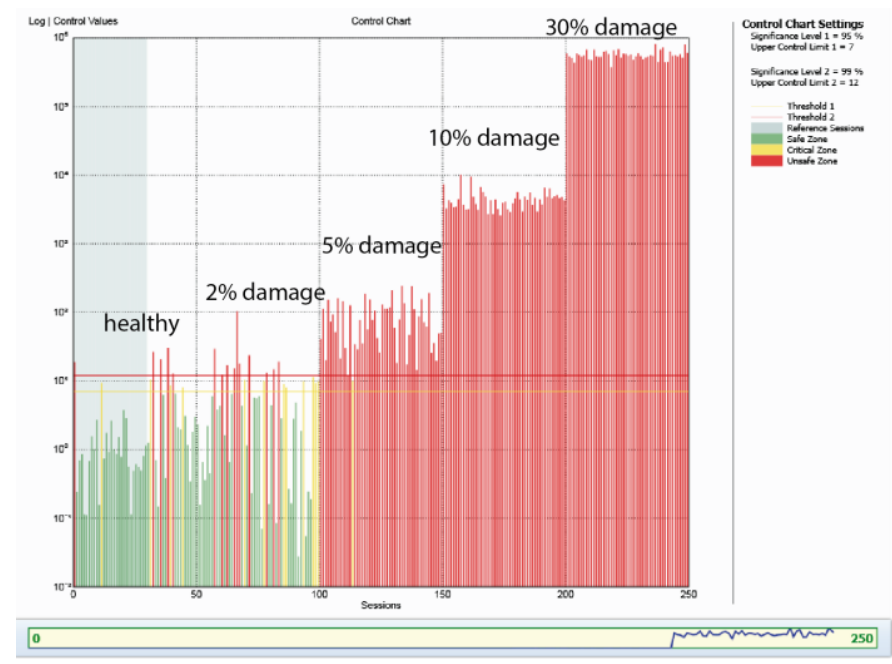

Figure 6. Hotelling $T^{2}$ control chart for simple system. Robust subspace and Mahalanobis-based detection. Case: noise $30 \%$.

Table 1. Comparison of the detection methods. Ability to detect damage in the simple system for different noise levels.

\begin{tabular}{|l|l|l|l|l|l|}
\hline \multicolumn{2}{|c}{ Case/Method } & \multicolumn{4}{c|}{ Damaged sets detected } \\
\cline { 3 - 6 } & 2\% damage & $\mathbf{5 \%}$ damage & $\mathbf{1 0 \%}$ damage & 30\% damage \\
\hline \multirow{2}{*}{ Noise 3\% } & Subspace-based & $100 \%$ & $100 \%$ & $100 \%$ & $100 \%$ \\
\cline { 2 - 6 } & Mahalanobis-based & $100 \%$ & $100 \%$ & $100 \%$ & $100 \%$ \\
\hline \multirow{2}{*}{ Noise 10\% } & Subspace-based & $10 \%$ & $26 \%$ & $100 \%$ & $100 \%$ \\
\cline { 2 - 6 } & Mahalanobis-based & $40 \%$ & $100 \%$ & $100 \%$ & $100 \%$ \\
\hline \multirow{2}{*}{ Noise 30\% } & Subspace-based & $2 \%$ & $8 \%$ & $100 \%$ & $100 \%$ \\
\cline { 2 - 6 } & Mahalanobis-based & $0 \%$ & $40 \%$ & $100 \%$ & $100 \%$ \\
\hline
\end{tabular}




\section{DOGNA BRIDGE}

For the general overview of the measurements, the first six singular values of the cross-spectral densities from the output acceleration data for the reference state are plotted in Figure 7.

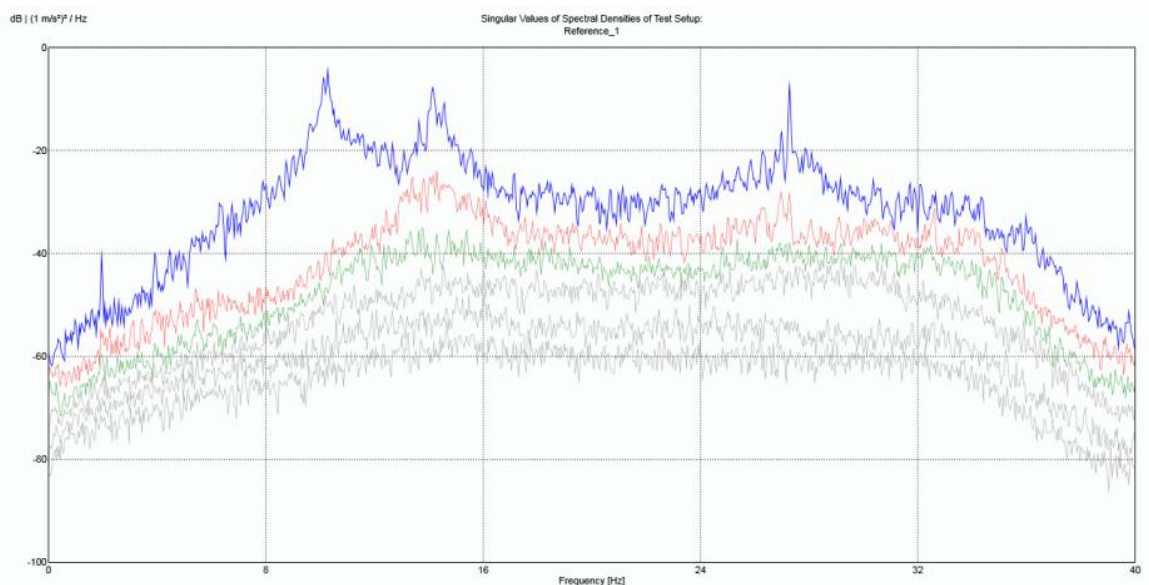

Figure 7. First six singular values of the cross spectral densities of the output acceleration data for the reference state.

In the frequency range of $0-40 \mathrm{~Hz}$ there are three visible modes based on the singular values, Figure 7 . Hence the damage detection does not involve the system identification, the modal properties won't be analyzed further.

The total number of 22 data sets are analyzed. The reference measurement model for the full scale example is calculated from 6 out of 8 healthy data records. The comparison of the subspace-based and Mahalanobis-based detection methods and the control chart combining the methods is illustrated in Figure 8.
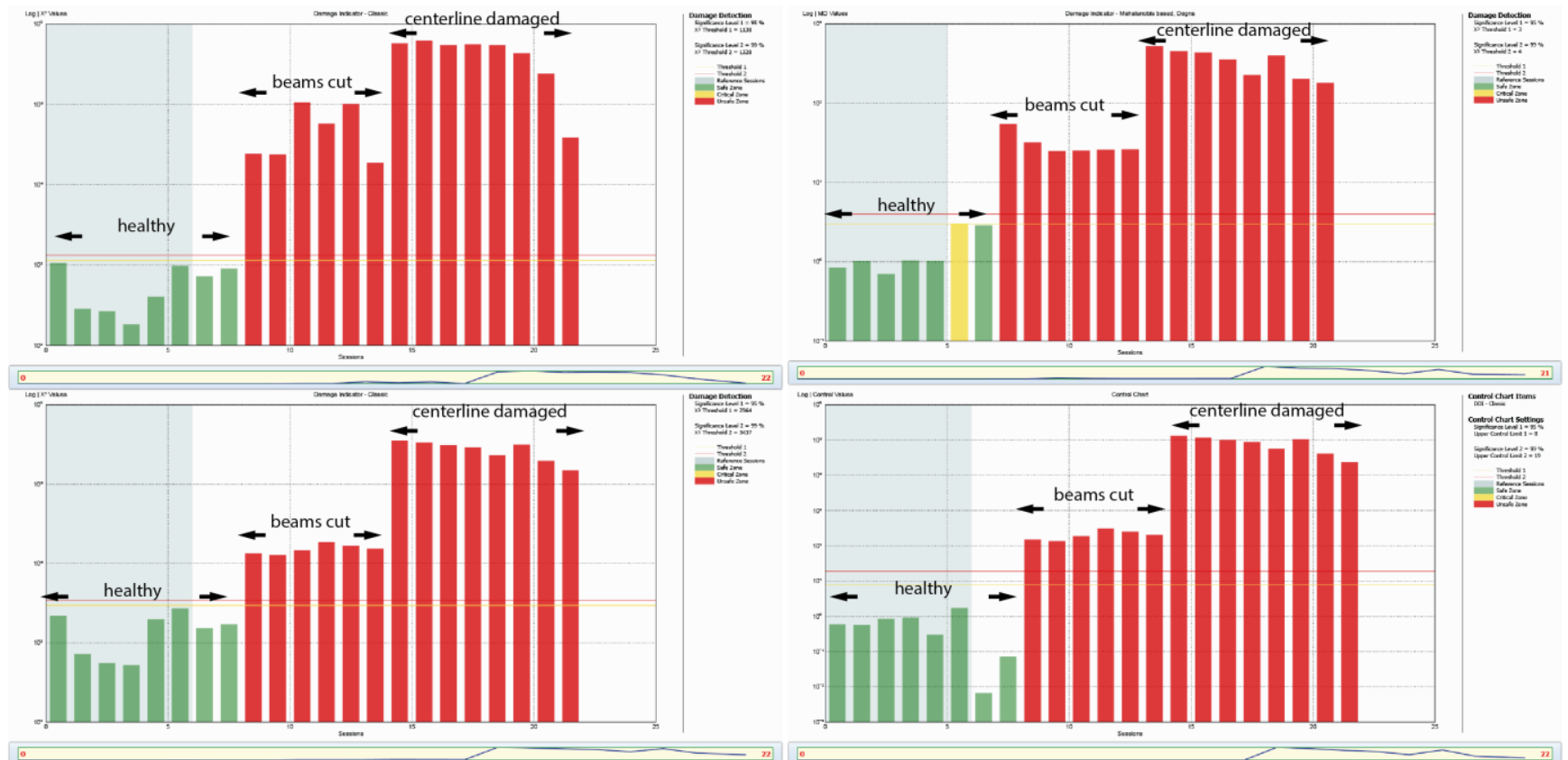

Figure 8. Damage detection in Dogna bridge. Damage indicators from top: Robust subspace (left). Mahalanobis-based (right). From bottom: Classical subspace (left), Hotelling control chart (right).

Both the subspace-based and Mahalanobis-based method detect the damages in all the test cases. The time stamp for the particular type of damage in the damaged data (number of cuts in the beams, damage of the centerline of the tested span) is unknow, hence the exact performance of single method is difficult to assess. However, each method clearly distinguish the healthy data from the damaged and detects the last stage of the campaign (the damage of the centerline of the span), see Figure 3. 


\section{CONCLUSIONS}

In this paper a recently developed Mahalanobis metric based damaged detection was presented and compared to well-known and proven subspace-based damage detection approaches. The methods were tested on the noisy simulation data and the full scale bridge example. Both test cases were excited by an ambient excitation with a changing intensity. Proposed Mahalanobisbased method gives clear damage indicator for both the simulation and full scale tests and performs effectively comparing to the subspace-based approach regarding the noisy simulations. The combination of the methods in the control chart was successful and resulted in the most effective detection of the damage both for simulations and full scale test.

The research regarding the detection methods will continue with focus on testing the algorithms on more extensive full scale experimental campaigns with a different types and extent of damages. Hence the fusion of the methods resulted in the most accurate picture of the damage it will be followed in a next work. The performance of empirical block Hankel matrices as a damage sensitive features will be questioned and compared with different transformation of the output data.

\section{REFERENCES}

[1] Farrar, C., Doebling, S., Nix, D. (2001). "Vibration-based structural damage identification" Philos. Trans. R. Soc. A Math. Phys. Eng. Sci. 359 (1778) 131-149.

[2] Doebling, S., Farrar, C., Prime, M. (1998). "A summary review of vibration-based damage identification methods". Shock Vib. Dig. 30 (2) 91-105

[3] Carden, E. and Fanning, P. (2004). "Vibration based condition monitoring: A review." Struct. Health Monit. 3 (4) 355377.

[4] Worden, K., Farrar, C., Manson, G., Park, G. (2007). "The fundamental axioms of structural health monitoring." Proc. R. Soc. A Math. Phys. Eng. Sci. 463 (2082) 1639-166

[5] Worden, K., Manson, G., Fieller, N. (2000). Damage detection using outlier analysis, J. Sound Vib. 229 (3) 647-667.

[6] Van Overschee, P., \& De Moor, B. (1996). Subspace identification for linear systems: Theory, implementation, applications. Kluwer.

[7] Balmès, E. , Basseville, M. , Mevel, L. , \& Nasser, H. (2009). Handling the temperature effect in vibration-based monitoring of civil structures: a combined sub- space-based and nuisance rejection approach. Control Engineering Practice, 17 (1), 80-87.

[8] Bernal, D. (2013). Kalman filter damage detection in the presence of changing process and measurement noise. Mechanical Systems and Signal Processing, 39 (1-2), 361-371.

[9] Döhler, M., \& Hille, F. (2014). Subspace-based damage detection on steel frame structure under changing excitation. In Proceedings of 32nd international modal analysis conference. Orlando, FL, USA.

[10]Döhler, M. , Hille, F. , Mevel, L. , \& Rücker, W. (2014). Structural health monitoring with statistical methods during progressive damage test of S101 Bridge. Engineering Structures, 69, 183-193.

[11]Döhler, M., \& Mevel, L. (2013). Subspace-based fault detection robust to changes in the noise covariances. Automatica, 49 (9), 2734-2743.

[12]Döhler, M., Mevel, L., \& Hille, F. (2014). Subspace-based damage detection under changes in the ambient excitation statistics. Mechanical Systems and Signal Processing, 45 (1), 207-224.

[13] Basseville, M., Abdelghani, M., \& Benveniste, A. (2000). Subspace-based fault detection algorithms for vibration monitoring. Automatica, 36 (1), 101-109.

[14]Zhou, Y., Maia, N.M., \& Wahab, M.A. (2016). Damage detection using transmissibility compressed by principal component analysis enhanced with distance measure. Journal of Vibration and Control 1-19.

[15] Cheung, A., Cabrera, C., Sarabandi, P., Nair, K.K., Kiremidjian, A. (2008). The application of statistical pattern recognition methods for damage detection to field data. Smart Material and Structures 17, 1-12.

[16] Filzmoser, P. (2004). A multivariate outlier detection method. In Proceedings of State University conference, 18

[17] ARTeMIS Pro. 5.1 (2016). Structural Vibration Solutions A/S. NOVI Science Park, DK-9220 Aalborg East Denmark.

[18]Lowry, C.A., Montgomery, D.C. (1995) A review of multivariate control charts. IIE Transactions, 27 (6) 800-810.

[19] Yan, A.M., Golinval, J.C. (2006) Null subspace-based damage detection of structures using vibration measurements. Mechanical Systems and Signal Processing 20, 611-626.

[20] Hardin, J., Rocke, D.M. (2005) The Distribution of Robust Distances. Journal of Computational and Graphical Statistics, 14 (4) 928-946. 